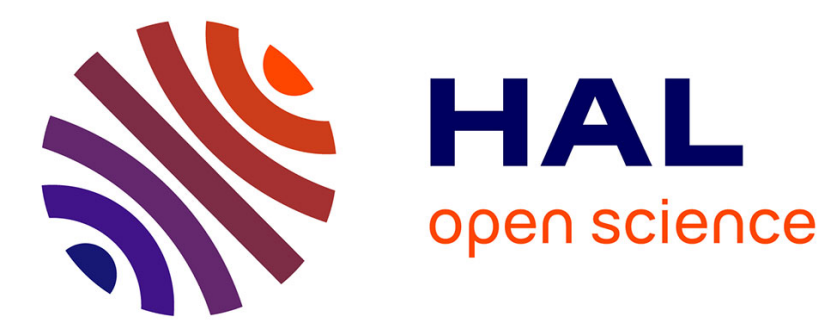

\title{
Generic differential kinematic modeling of articulated multi- monocycle mobile robots
}

\author{
Frédéric Le Menn, Philippe Bidaud, Faiz Ben Amar
}

\section{To cite this version:}

Frédéric Le Menn, Philippe Bidaud, Faiz Ben Amar. Generic differential kinematic modeling of articulated multi- monocycle mobile robots. IEEE International Conference on Robotics and Automation (ICRA 2006), May 2006, Orlando, FL, United States. pp.1505-1510, 10.1109/ROBOT.2006.1641921. hal-03178083

\section{HAL Id: hal-03178083 \\ https://hal.science/hal-03178083}

Submitted on 24 Mar 2021

HAL is a multi-disciplinary open access archive for the deposit and dissemination of scientific research documents, whether they are published or not. The documents may come from teaching and research institutions in France or abroad, or from public or private research centers.
L'archive ouverte pluridisciplinaire HAL, est destinée au dépôt et à la diffusion de documents scientifiques de niveau recherche, publiés ou non, émanant des établissements d'enseignement et de recherche français ou étrangers, des laboratoires publics ou privés. 


\title{
Generic differential kinematic modeling of articulated multi-monocycle mobile robots
}

\author{
Frederic Le Menn, Philippe Bidaud, Faiz Ben Amar \\ Universite Pierre et Marie Curie - Paris 6 \\ Laboratoire de Robotique de Paris, CNRS FRE 2507 \\ 92265 Fontenay aux Roses, France \\ Email : \{lemenn, bidaud, amar $\} @$ robot.jussieu.fr
}

\begin{abstract}
This paper presents a generic kinematic modeling approach for articulated multi-monocycle mobile robots. The formulation proposed to deduce the input/output velocity equations for such kinematic structures is an extension of the reciprocal screw based method of asymmetrical and constrained parallel mechanisms. The efficiency of this methodology for setting up the differential kinematic model is illustrated through a application : the robuROC6 mobile robot. Its complex kinematic structure is first transformed into a spatial parallel mechanism which encapsulates the differential driving wheels system. Then, the analytical form of the reciproqual screw system which corresponds to the actively controlled wrenches applied on the controlled body is established. Reciprocally, it describes the way the wheel velocities are transfered to the output body. It also provides a geometrical information for an exhaustive singularity analysis and traction distribution optimization during the evolution of the system on highly irregular surfaces. From the differential kinematic model, the concept of traction ellipsoid is introduced for evaluating quantitatively the obstacle clearance capabilities when the configuration of the system and the contact conditions are highly variable.
\end{abstract}

\section{INTRODUCTION}

RobuROC6 (shown in figure 1) has been designed in a collaboration between the ROBOSOFT Inc [1] and the Laboboratoire de Robotique de Paris in response to a Research Program launched in 2004 by the French Defense Agency (DGA - Délégation Générale l'Armement) called MiniRoc whose aim is to develop and evaluate experimentally several semi-autonomous system serving as an extension of the human soldier. RobuROC6 belongs to this class of robot vehicle named Tactical Mobile Robot (TMR). TMRs are basically high mobility small vehicles supposed to operate in highly uncertain urban outdoor/indoor environments. TMR development did not truly begin until the early 1990s. Until then, the military's primary focus for ground robotics was on the development of Unmanned Ground Vehicles (UGVs). Nevertheless, various families of TMRs have been developed during the last decade. Their design is more compact and robust than exploration robotics vehicles and have to satisfy specific operational requirements (see [2]). One of the main features of robuROC6 is its ability to operate in extremely rough terrain and negotiating stairs (circular stairs) or clear obstacles with height greater than its wheel radius. Moreover, its concept was designed to offer reconfiguration capabilities

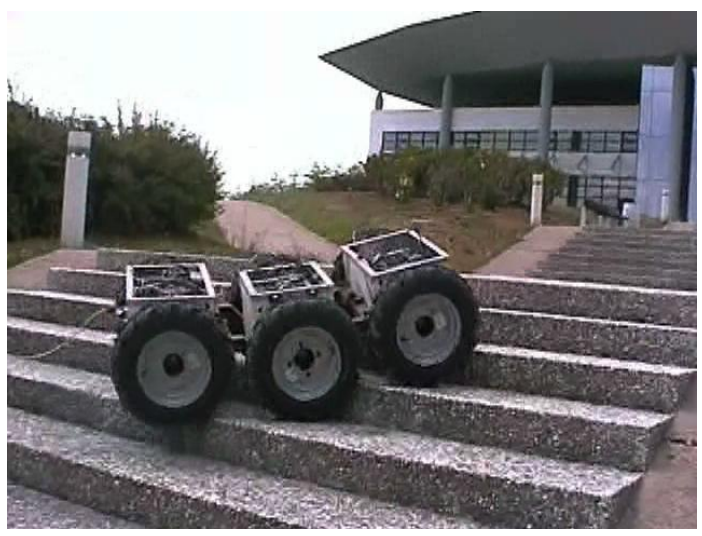

Fig. 1. The RobuRoc mobile robot climbing a stair

for providing either a maximum of ground adaptation for traction optimization or a high manoeuvrability. RobuROC6 kinematics can be considered as a series of 3 unicycle modules linked together by two orthogonal rotoid passive joints (a roll and a pitch motion in between the modules). It has been optimized for stair-climbing as well as several typical bumps and jumps clearance.

The differential kinematic model plays a fundamental role in the robot performance analysis (mobility, input/output velocity transmission, singularities, traction transmission, etc ...), odometry and trajectory tracking control. The method for deriving the input/output velocity relationship, which is widely employed for wheeled mobile robots, consists of introducing geometrical transformations between the moving bodies and their derivative to obtain equation of motion both by assuming ideal rolling conditions as closed-loop constraints [3]. Systematic formulations have been developed for various combinations of driving and steering wheels [4] [5]. Realistic sliding models in the soil/wheel interaction have also be introduced for developing more complete models [6] [7] [8]. For eliminating the passive joint variables in the kinematic equations and obtain the closed form of the input/output velocity relationship, a particular procedure which is generally system dependent has to be found.

The particular contribution of the paper is a generic and efficient method for obtaining very efficient kinematic model for complex articulated locomotion systems such as robuROC6. 
The proposed method is based on the use of reciproqual screws. Screw-based approaches have been employed as a general framework for the mobility analysis of various types of simple planar mobile robots [9]. Here, we consider passively articulated multi-monocycle mobile robots evolving on 3D surfaces. An equivalent kinematic model which encapsulate monocycle sub-system kinematics is defined. Hence, the articulated multi-monocycle structure is represented as a constrained asymmetric parallel system. A reciproqual screwbased approach can be then developed to obtain the input/output instantaneous velocities equations. Moreover, this approach gives a better geometrical insight into the problem of singularities and more generally into motion and force transmission characteristics of the system. The concept of traction ellipsoid is introduced for evaluating quantitatively the obstacle clearance capabilities when the configuration of the system and the contact conditions are constantly changing.

\section{KinEMATIC MODELING AND ANALYSIS}

\section{A. Kinematic description}

RobuROC6 is an articulated wheeled robot vehicle designed for use in urban environments (open spaces such as city streets and building interiors). It must have the ability to overcome obstacles such as rubble piles, pipes, railroad tracks, etc ... and to climb up straight and circular stairs. RobuROC6 is composed of a multi-monocycle whose kinematic structure as depicted in figure 4 . It consists of a central pod ${ }^{1}$ and two identical pods. Each pod is steered and driven by two actuated conventional wheels on which a lateral slippage may occur. The rear and the front pods are symmetrically arranged about the central pod. They are attached to this later one by two orthogonal passive rotoid joints providing a roll/pitch relative motion for keeping the wheels on the ground to maintain traction of the pod while traversing irregular surfaces. This kinematic design permits to transform robuROC6 in a 4-wheel configuration as shown in figure 2.

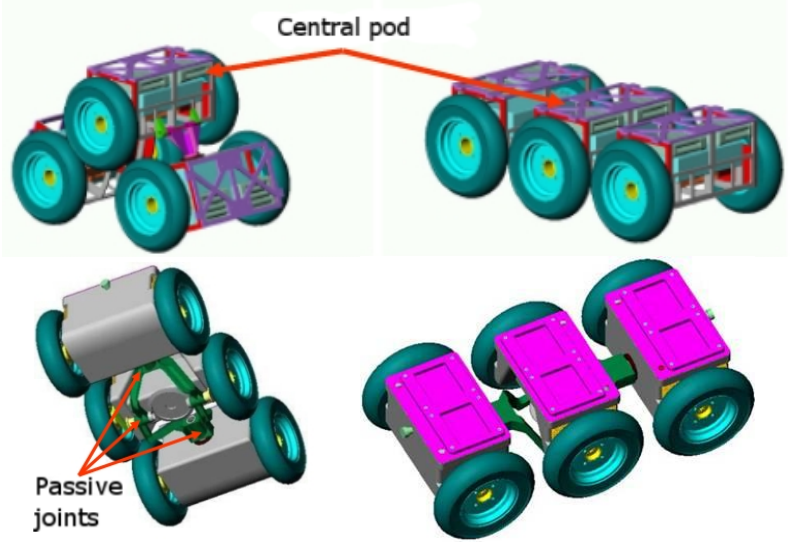

Fig. 2. RobuROC6 in a 4-wheel and 6-wheel configurations and details of its articulations

\footnotetext{
${ }^{1}$ term introduced to define a monocycle element
}

\section{B. Kinematic modeling}

Kinematics plays a fundamental role in design, dynamic modeling, and control. In this section, we illustrate a methodology for modeling and analysis of articulated multi-monocycle mobile robots. The relationship between the central pod velocity in a reference frame and wheel velocity vector can be greatly simplified by extending the methodology used for parallel mechanisms [10] to asymmetric constrained mechanisms. A parallel manipulator typically consists of several limbs, made up of an open loop mechanism, connecting a moving platform to the ground. Here, the body $S_{0}$ of central module can be seen as the moving platform. It is connected to the ground by a differential steering system as well as by the rear and the front modules via two passive revolute joints which can be seen as 2 others "limbs" connecting $S_{0}$ to the ground. Hence, the whole mechanism is assimilable to an asymmetric constrained mechanism with a mobility equal to 4 .

Jacobian of a module: Each individual module $j(j=0$ for the central, $j=1,2$ for the others) can be modeled as an equivalent serial open-chain mechanism (see figure 3 ).

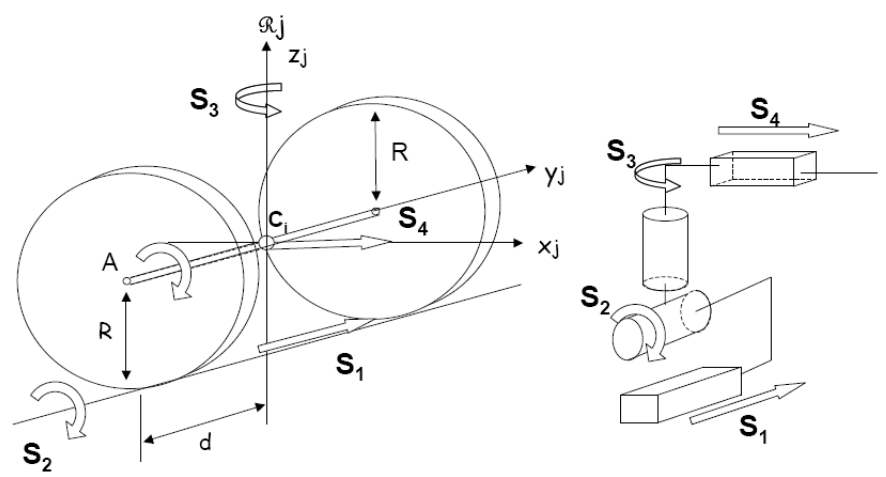

Fig. 3. The differential driving wheels mechanism (left) and its equivalent open-chain mechanism (right)

The $j^{t h}$ differential driving wheels mechanism kinematics can be represented by a set of four unit instantaneous twists $\hat{\$}_{i}^{j}=\left(s_{i}^{j}, s_{0 i}^{* j}=r_{i}^{j} \times s_{i}^{j}+\mu_{i}^{j} s_{i}^{j}\right)^{t} i=1,4$ where $s_{i}^{j}$ is a unit vector along the direction of the screw axis, $r_{i}^{j}$ is the position vector of any point of the screw axis with respect to a reference point $O$ and $\mu_{i}$ is the pitch of the screw. The normalized Plucker coordinates of these screws written at the axle middle point of the central axle $C_{j}$ and in the basis of the $\mathcal{R}_{j}$ local frame form the Jacobian of a monocycle $J_{m}^{j}$ is as follows :

$$
J_{m}^{j}=\left\{\hat{\$}_{1}^{j}, \hat{\$}_{2}^{j}, \hat{\$}_{3}^{j}, \hat{\$}_{4}^{j}\right\}=\left(\begin{array}{cccc}
0 & 0 & 0 & 0 \\
0 & 1 & 0 & 0 \\
0 & 0 & 1 & 0 \\
0 & a_{j} & 0 & 1 \\
1 & 0 & 0 & 0 \\
0 & 0 & 0 & 0
\end{array}\right)
$$


The reciprocal complement $W^{j}$ in $s e(3)$ of the system $J_{m}^{j}$ has a dimension 2. It represents the natural constraints acting on the system due to the wheel/soil constraints :

$$
W^{j}=\left(\begin{array}{cc}
0 & 0 \\
0 & 0 \\
0 & 1 \\
1 & 0 \\
0 & 0 \\
0 & 0
\end{array}\right)
$$

For the module $j$, the amplitude $\omega_{z_{j}}$ and $v_{x_{j}}$ of the screws $\hat{\$}_{3}^{j}$ and $\hat{\$}_{4}^{j}$ are related to left and right differentially driven wheel velocities $\left(\dot{\theta}_{j 1}, \dot{\theta}_{j 2}\right)$ by the relationship:

$$
\left(\begin{array}{c}
\omega_{z j} \\
v_{z j}
\end{array}\right)=1 / 2\left(\begin{array}{cc}
R / d & -R / d \\
R & R
\end{array}\right)\left(\begin{array}{c}
\dot{\theta}_{1 j} \\
\dot{\theta}_{2 j}
\end{array}\right)
$$

or in a compact matrix form:

$$
\dot{q}^{j}=J_{a}^{j} \dot{\theta}^{j}
$$

where $R$ is the radius of the wheels and $d$ is the half length of the width of unicycle module. $J_{a}^{j}$ is the Jacobian matrix of the active part of the pod mechanism.
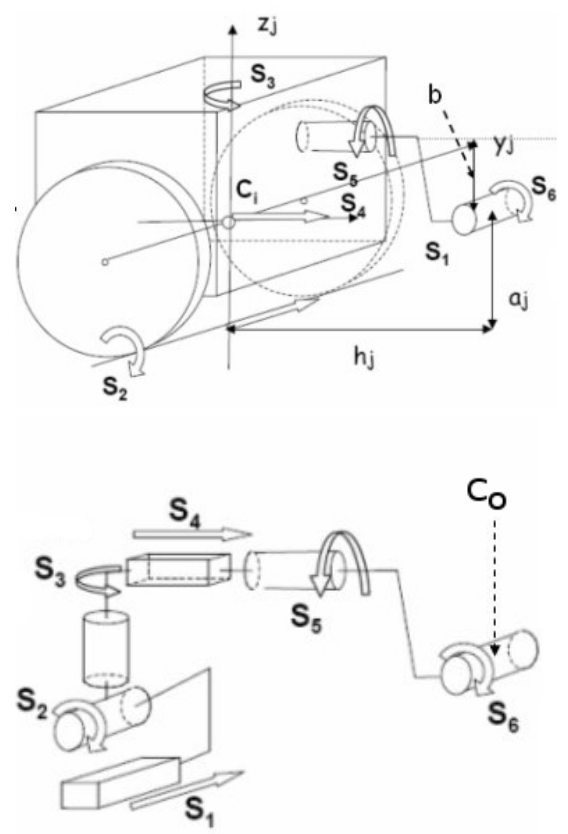

Fig. 4. The differential driving wheels mechanism (left) and its equivalent open-chain mechanism (right) (front/rear module)

\section{Reciprocal Screws of the 3 limbs}

Using the notation introduced in figure 4, the instantaneous twist $\$_{P}$ of the central body $\left(S_{0}\right)$ with respect to the ground can be expressed as a linear combination of the $n$ actuated and non-actuated joints screws of each $j$ sub-chain:

$$
\$_{P}=\sum_{i=1}^{n} \dot{q}_{i}^{j} \hat{\$}_{i}^{j} \text { for } j=0,1,2
$$
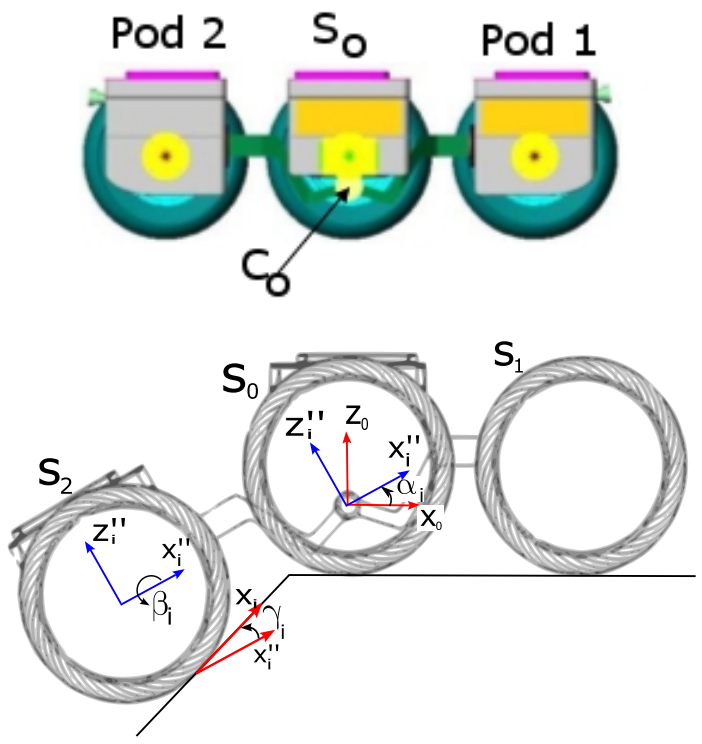

Fig. 5. Definition of $\alpha_{i}, \beta_{i}$ and $\gamma_{i}$ angles.

where $\dot{q}_{i}^{j}$ and $\hat{\$}_{i}^{j}$ denote the intensity and the unit screw associated with the $i$ th joint of the $j$ th limb. By considering alternatively the $j=0,1,2$ (which denotes respectively the labels for the central, front and rear pods), we obtain:

$$
\begin{gathered}
\$_{P}=\dot{q}_{1}^{0} \hat{\$}_{1}^{0}+\dot{q}_{2}^{0} \hat{\$}_{2}^{0}+\omega_{z_{0}}^{0} \hat{\$}_{3}^{0}+v_{x_{0}}^{0} \hat{\$}_{4}^{0} \\
\$_{P}=\dot{q}_{1}^{1} \hat{\$}_{1}^{1}+\dot{q}_{2}^{1} \hat{\$}_{2}^{1}+\omega_{z_{1}}^{1} \hat{\$}_{3}^{1}+v_{x_{1}}^{1} \hat{\$}_{4}^{1}+\dot{q}_{5}^{1} \hat{\$}_{5}^{1}+\dot{q}_{6}^{1} \hat{\$}_{6}^{1} \\
\$_{P}=\dot{q}_{1}^{2} \hat{\$}_{1}^{2}+\dot{q}_{2}^{2} \hat{\$}_{2}^{2}+\omega_{z_{2}}^{2} \hat{\$}_{3}^{2}+v_{x_{2}}^{2} \hat{\$}_{4}^{2}+\dot{q}_{5}^{2} \hat{\$}_{5}^{1}+\dot{q}_{6}^{1} \hat{\$}_{6}^{1}
\end{gathered}
$$

Equations (4) (5) (6) contain many unactuated joint rates $(2 \times 3+4)$ that must be eliminated to obtain a relationship between the instantaneous screw which defines the absolute motion $\$_{P}$ of the central body and the wheel's velocities vector $\dot{\theta}$. This can be done very efficiently by using a set of reciprocal screws $\$_{i}^{r j}$ which is by definition reciproqual to all screws of the $j^{\text {th }} \operatorname{limb}$ except the actuated joint of order $i$ in the equivalent open chain. Two screws $\hat{\$}_{1}$ and $\hat{\$}_{2}$ are considered to be reciprocal if they satisfy the condition:

$$
s_{1} . s_{02}^{*}+s_{2} . s_{01}^{*}=0
$$

This reciprocity condition can be stated if the virtual work between a wrench and a twist is equal to zero. A detailed description of screw systems can be found in [11].

The Jacobian of the $j=1,2$ equivalent open chain formed by a monocycle and the two passive revolute joints expressed at the point $C_{0}$ and in the local $\mathcal{R}_{j}$ (see figure 5) are $\left({ }^{2}\right.$ ):

$$
J_{j}=\left(\begin{array}{cccccc}
0 & 0 & 0 & 0 & C \gamma_{j} & S \beta_{j} S \gamma_{j} \\
0 & 1 & 0 & 0 & 0 & C \beta_{j} \\
0 & 0 & 1 & 0 & S \gamma_{j} & -S \beta_{j} C \gamma_{j} \\
0 & a_{j} & 0 & 1 & b S \beta_{j} S \gamma_{j} & 0 \\
1 & 0 & h_{j} & 0 & b C \beta_{j} & 0 \\
0 & -h_{j} & 0 & 0 & -b S \beta_{j} C \gamma_{j} & 0
\end{array}\right)
$$$$
{ }^{2} C \equiv \cos , S \equiv \sin \text { and } T \equiv \tan
$$ 
These screws form a 6 -system provided fixed so that they are linearly independent. Hence, $\$_{3}^{r^{j}}$ and $\hat{\$}_{4}^{r^{j}}$ that are reciprocal to all screws except for respectively $\hat{\$}_{3}^{r^{j}}$ and $\hat{\$}_{4}^{r^{j}}$ for $j=1,2$ are unique. Their normalized Pluker coordinates $\left(u_{i}^{j}, v_{i}^{j}, w_{i}^{j}, l_{i}^{* j}, m_{i}^{* j}, n_{i}^{* j}\right)^{t}$ are respectively :

$$
\hat{\$}_{3}^{r^{j}}=\left[0,0,1, l_{3}^{* j}, h_{j}, n_{3}^{* j}\right]^{t} \text { for } \mathrm{j}=1,2
$$

with :

$$
\begin{aligned}
l_{3}^{* j} & =b C^{2} \gamma_{j} S \beta_{j}-h_{j} T^{-1} \beta_{j} S \gamma_{j} \\
n_{3}^{* j} & =C \gamma_{j}\left(b S \gamma_{j} S \beta_{j}+h_{j} T^{-1} \beta_{j}\right)
\end{aligned}
$$

$\hat{\$}_{3}^{r^{j}}$ is a non-null pitch reciproqual screw. The pitch $\mu_{3}^{j}=n_{3}^{* j}$ which turns to infinity when $\beta_{j}=0$.

$$
\hat{\$}_{4}^{r^{j}}=\left[u_{4}^{j}, 0, w_{4}^{j}, l_{4}^{* j}, m_{4}^{* j}, 0\right]^{t} \text { for } \mathrm{j}=1,2
$$

with :

$$
\begin{aligned}
& u_{4}^{j}=U_{4}^{j} /\left[\left(U_{4}^{j}\right)^{2}+\left(W_{4}^{j}\right)^{2}\right]^{1 / 2} \\
& w_{4}^{j}=W_{4}^{j} /\left[\left(U_{4}^{j}\right)^{2}+\left(W_{4}^{j}\right)^{2}\right]^{1 / 2}
\end{aligned}
$$

$$
l_{4}^{* j}=b C \beta_{j} S \beta_{j}\left(a_{j} C \gamma_{j}-h_{j} S \gamma_{j}\right) /\left[\left(U_{4}^{j}\right)^{2}+\left(W_{4}^{j}\right)^{2}\right]^{1 / 2}
$$

$m_{4}^{* j}=-b S^{2} \beta_{j} S \gamma_{j}\left(a_{j} C \gamma_{j}-h_{j} S \gamma_{j}\right) /\left[\left(U_{4}^{j}\right)^{2}+\left(W_{4}^{j}\right)^{2}\right]^{1 / 2}$

and

$$
\begin{gathered}
U_{4}^{j}=h_{j} C \beta_{j} C \gamma_{j}+b S^{2} \beta_{j} S \gamma_{j} C \gamma_{j} \\
W_{4}^{j}=a_{j} C \beta_{j} C \gamma_{j}+b S^{2} \beta_{j} S^{2} \gamma_{j}
\end{gathered}
$$

$\hat{\$}_{4}^{r^{j}}$ is a force located in the plane $\left(x_{j}, z_{j}\right)$ passing through the point $C_{0}$ when $\beta_{j}=\gamma_{j}=0$.

The reciprocal screws $\hat{\$}_{3}^{r^{0}}$ and $\hat{\$}_{4}^{r^{0}}$ have to be determined by considering the $n=4$-system $\left\{\hat{\$}_{1}^{0}, \hat{\$}_{2}^{0}, \hat{\$}_{3}^{0}, \hat{\$}_{4}^{0}\right\}$ of feasible motions. To treat constrained mechanisms $(n<6)$, as mentioned in [12] the screw-based approach have to be adapted. An "actively" applicable wrench basis for the constrained system can be first determined by using a projection of any reciproqual screw coordinate vector (the screw of the wrench) onto the orthogonal complement $\left(W^{j}\right)^{\perp}$ of the natural constraints 2system $\left(W^{j}\right)$ :

$$
\left(W^{j}\right)^{\perp}=\left(\begin{array}{cccc}
1 & 0 & 0 & 0 \\
0 & 1 & 0 & 0 \\
0 & 0 & 0 & 0 \\
0 & 0 & 0 & 0 \\
0 & 0 & 1 & 0 \\
0 & 0 & 0 & 1
\end{array}\right)
$$

Then, the reciproqual screws will always have two of its components equal to zero :

$$
\left(f_{x}, f_{y}, 0,0, m_{y}, m_{z}\right)^{t}=\left(W^{j}\right)^{\perp}\left(f_{x}, f_{y}, f_{z}, m_{x}, m_{y}, m_{z}\right)^{t}
$$

In this reduced wrench space, the reciproqual screws $\hat{\$}_{3}^{r^{0}}$ and $\hat{\$}_{4}^{r^{0}}$ are unique since the columns of $J_{m}^{0}$ are linearly independent. In $C_{0}, h_{0}=0$ and their expression reduced to :

$$
\begin{aligned}
& \hat{\$ r}_{3}^{0}=[0,0,0,0,0,1]^{t} \\
& \hat{\$^{r}}{ }_{4}^{0}=\left[1,0,0,0, a_{0}, 0\right]^{t}
\end{aligned}
$$

The coordinates of these reciprocal screws can be directly computed by multiplying (7) and (8) in the $\mathcal{R}_{0}$ frame by using the block matrix:

$$
E_{0 j}=\left(\begin{array}{cc}
R_{j 0}^{t} & 0_{3 \times 3} \\
0_{3 \times 3} & R_{j 0}^{t}
\end{array}\right)
$$

with :

$R_{j 0}=\left(\begin{array}{ccc}C \alpha_{j} C \gamma_{j}-S \alpha_{j} C \beta_{j} S \gamma_{j} & S \beta_{j} S \gamma_{j} & -S \alpha_{j} C \gamma_{j}-C \alpha_{j} C \beta_{j} S \gamma_{j} \\ S \alpha_{j} S \beta_{j} & C \beta_{j} S \beta_{j} & C \beta_{j} S \gamma_{j}+S \alpha_{j} C \beta_{j} C \gamma_{j} \\ C S \beta_{j} C \gamma_{j} & -S \alpha_{j} S \gamma_{j}+C \alpha_{j} C \beta_{j} C \gamma_{j}\end{array}\right)$

Input/Output velocity equation

By multiplying (via the reciprocal screw product) each side of the equations (4) (5) (6) with the associated reciprocal screws, it produces a set of equations which can be written in a matrix form as follows:

$$
B \dot{q}_{a}=D \dot{X}
$$

with $\dot{q}_{a}=\left(\omega_{z_{1}}^{1}, v_{x_{1}}^{1}, \omega_{z_{0}}^{0}, v_{x_{0}}^{0}, \omega_{z_{2}}^{2}, v_{x_{2}}^{2}\right)^{t}, \omega_{z_{j}}^{j}$ and $v_{x_{j}}^{j}$ denoting respectively the linear and angular velocities produced by the differential steering system of the pod $j$ along $x_{j}$ and about $z_{j}, \dot{X}=\left(\bar{w}_{x 0}, \bar{w}_{y 0}, \bar{w}_{z 0}, \bar{v}_{x 0}, \bar{v}_{y 0}, \bar{v}_{z 0}\right)^{t} B$, the $(6 \times 6)$ matrix which multiply the active joint rates is a diagonal matrix whose components are:

$$
\begin{gathered}
B=\operatorname{diag}\left(\hat{\$}_{3}^{r 1} \cdot \hat{\$}_{3}^{1}, \hat{\$}_{4}^{r 1} \cdot \hat{\$}_{4}^{1}, \hat{\$}_{3}^{r 0} \cdot \hat{\$}_{3}^{0}, \hat{\$}_{4}^{r 0} \cdot \hat{\$}_{4}^{0}, \hat{\$}_{3}^{r 2} \cdot \hat{\$}_{3}^{2}, \hat{\$}_{4}^{r 2} \cdot \hat{\$}_{4}^{2}\right) \\
B=\operatorname{diag}\left(n_{3}^{1}, u_{4}^{1}, n_{3}^{0}, u_{4}^{0}, n_{3}^{2}, u_{4}^{2}\right)
\end{gathered}
$$

and $D$ is a $(6 \times 6)$ matrix whose lines are the reciprocal screws coordinates in the frame $\mathcal{R}_{0}$ :

$$
D=\left(\begin{array}{cccccc}
(0 & 0 & 1 & l_{3}^{* 1} & h_{1} & \left.n_{3}^{* 1}\right) E_{01}^{t} \\
\left(u_{4}^{1}\right. & 0 & w_{4}^{1} & l_{4}^{* 1} & m_{4}^{* 1} & 0) E_{01}^{t} \\
(0 & 0 & 0 & 0 & 0 & 1) \\
(1 & 0 & 0 & 0 & a_{0} & 0) \\
(0 & 0 & 1 & l_{3}^{* 2} & h_{2} & \left.n_{3}^{* 2}\right) E_{02}^{t} \\
\left(u_{4}^{2}\right. & 0 & w_{4}^{2} & l_{4}^{* 2} & m_{4}^{* 2} & 0) E_{02}^{t}
\end{array}\right)
$$

By observation of matrix $D$ it can be deduced that the lateral velocity of the system is not controlled kinematically. By introducing the block matrix $J_{a}(3)$ :

$$
J_{a}=\left(\begin{array}{c|c|c}
J_{a_{1}} & 0 & 0 \\
\hline 0 & J_{a_{0}} & 0 \\
\hline 0 & 0 & J_{a_{2}}
\end{array}\right)
$$

we obtain the kinematic control model of the vehicle :

$$
J_{a} \dot{\theta}=D \dot{X}
$$

where

$$
\dot{\theta}=\left(\dot{\theta}_{11}, \dot{\theta}_{12}, \dot{\theta}_{01}, \dot{\theta}_{02}, \dot{\theta}_{21}, \dot{\theta}_{22}\right)^{t}
$$




\section{OBstaCle CLEARANCE CAPACITIES}

For simplicity, we will restrict the analysis to the two dimensional case of the obstacle clearance : $\beta_{j}=0$. The reciprocal screws become :

$$
\begin{aligned}
& \hat{\$}_{3}^{j}=\left[\begin{array}{llllll}
0 & 0 & 0 & 0 & 0 & 1
\end{array}\right]^{t} \\
& \hat{\$}_{4}^{j}=\left[\begin{array}{llllll}
C \lambda_{j} & 0 & S \lambda_{j} & 0 & 0 & 0
\end{array}\right]^{t}
\end{aligned}
$$

with :

$$
\begin{gathered}
C \lambda_{j}=h_{j} / \sqrt{a_{j}^{2}+h_{j}^{2}} \\
S \lambda_{j}=a_{j} / \sqrt{a_{j}^{2}+h_{j}^{2}} \\
B=\operatorname{diag}\left(1, C \lambda_{1}, 1,1,1, C \lambda_{2}\right) \\
D=\left(\begin{array}{ccccccc}
0 & 0 & 0 & 0 & 0 & 1 \\
C \varphi_{1} & 0 & S \varphi_{1} & 0 & 0 & 0 \\
0 & 0 & 0 & 0 & 0 & 1 \\
1 & 0 & 0 & 0 & a_{0} & 0 \\
0 & 0 & 0 & 0 & 0 & 1 \\
C \varphi_{2} & 0 & S \varphi_{2} & 0 & 0 & 0
\end{array}\right)
\end{gathered}
$$

with $\varphi_{j}=\alpha_{j}+\lambda_{j}+\gamma_{j}$.

This model clearly shows that the degree of mobility of the system in this particular case is equal to 4 . The lateral velocity of the system is not controlled kinematically as well as the rotation about the $x_{0}$ axis. It also describes how a vertical motion $\left(\bar{v}_{z_{0}}\right)$ of the central pod can be achieved to transform the system in a 4-wheel configuration. We obtain the kinematic control model of the vehicle :

$$
J_{a} \dot{\theta}=\left(\begin{array}{cccc}
0 & 0 & 0 & 1 \\
1 & t g \varphi_{1} & 0 & 0 \\
0 & 0 & 0 & 1 \\
1 & 0 & a_{0} & 0 \\
0 & 0 & 0 & 1 \\
1 & t g \varphi_{2} & 0 & 0
\end{array}\right)\left(\begin{array}{c}
\bar{v}_{x 0} \\
\bar{v}_{z 0} \\
\bar{\omega}_{x 0} \\
\bar{\omega}_{z 0}
\end{array}\right)=D \dot{X}
$$

On the other hand, force-moment transmission on the central body is reflected by the $D$ matrix. Its lines represent the elementary actions (here in the reduced wrench space $\left.\left(f_{x_{0}}, f_{z_{0}}, m_{y_{0}}, m_{z_{0}}\right)\right)$ developed by the driving torques $\left(\tau_{11}, \tau_{12}, \tau_{01}, \tau_{02}, \tau_{21}, \tau_{22}\right)^{t}$ on the central body. Similarly as when multiple robots exert forces or carry an object in cooperative way, $D$ is equivalent to $G^{t}, G$ representing the grasp matrix that contains the contact distribution and the way the active forces are transmitted throughout the central body. $G$ can be partitioned into 2 blocks $G=\left(G_{f} G_{m}\right)^{t}, G_{f}$ and $G_{m}$ representing the force and the moment transmission respectively. It is interesting to be able to compare the traction capabilities for different contact conditions. Hence, the set of forces and moments realizable by $\tau$ such $\|\tau\| \leq 1$ form an ellipsoid. A representative measure $\sigma$ of the traction derived from the image of this unit ball of active joint torques:

$$
\sigma=\left[\operatorname{det}\left(G_{f}^{t} G_{f}\right)\right]^{1 / 2}
$$

When $\beta_{j}=0(j=1,2)$, the traction index $\sigma$ is:

$$
\sigma=\left[\left(S \varphi_{1} C \varphi_{2}-C \varphi_{1} S \varphi_{2}\right)^{2}+S \varphi_{1}^{2}+S \varphi_{2}^{2}\right]^{1 / 2}
$$

This index is equal to zero when $G_{f}$ is singular. Then the force transmission in the vertical direction becomes null, this happens in configurations where $\gamma_{j}=0(j=1,2)$.
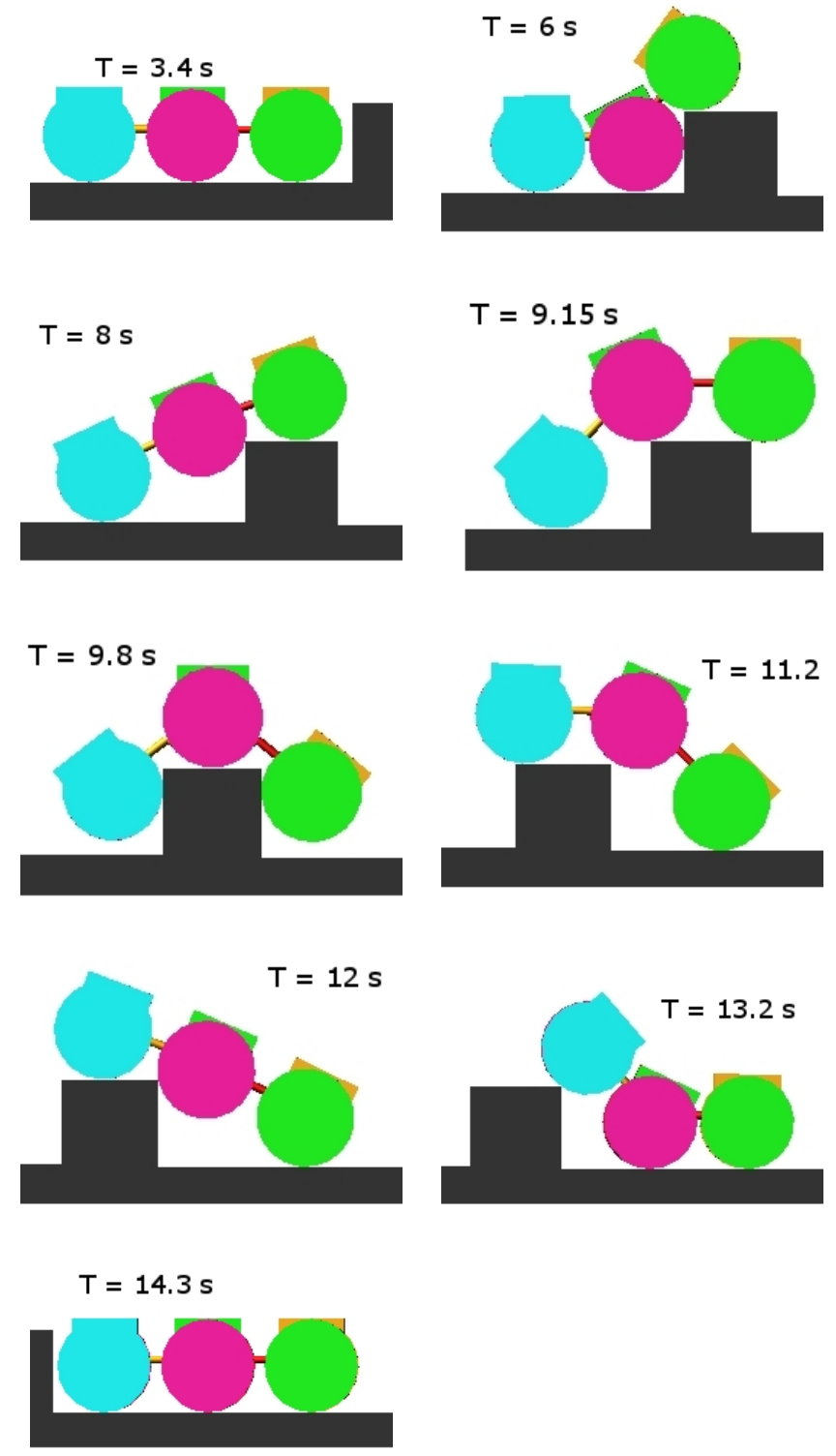

Fig. 6. Step clearance for robuROC6

To show the evolution of the manipulability index $\sigma$, we have studied the case of a step clearance for robuROC6. Figure 6 illustrates the evolution of the system during a step clearance while figure 7 shows the correlation between the geometry of the system and the manipulability index. The two singular values $\sigma_{1}$ and $\sigma_{2}$ correspond, in figure 7 , to the traction along the two principal axes of the manipulability ellipsoid. The singular value $\sigma_{1}$ represents the traction along the axis $x_{0}$ and $\sigma_{2}$ the traction along the axis $z_{0}$ in the $\mathcal{R}$, local frame. The curve is composed of three parts where each one contains a peak. Each part represents the evolution of the central pod : 


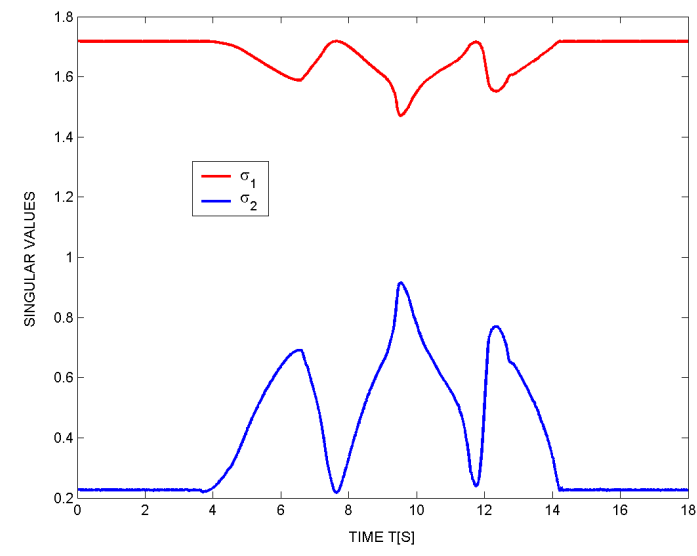

Fig. 7. Singular values $\sigma_{1}$ and $\sigma_{2}$ for step clearance

- the front and the rise of the step;

- on the step;

- the decrease and the rear of the step.

In all the simulation, the traction $\sigma_{1}$ is most important than $\sigma_{2}$. Indeed, a velocity setting along the $x_{0}$ axis is imposed. It is interesting to observe the evolution of the traction $\sigma_{2}$ during the simulation of a step clearance on figure 7 .

During the planar evolution of robuROC6 $([0 s ; 4 s])$, the participation of $\sigma_{2}$ is bare.

The time interval $[4 s ; 8 s]$ corresponds to the climbing of the step (figure 6). In the first part of this period, the central pod is pulled by the front one and pushed by the rear one (increase of $\left.\sigma_{2}\right)$. In the second part of the interval, the participation of the front pod during the climbing decreases while $\sigma_{1}$ increases. The time interval $[8 s ; 12 s]$ corresponds to the configuration in which the central pod is on the step (figure 6). In the same time, the central pod is pushed by the rear one and pulled by the front pod to produce an horizontal movement. These actions produce, first, an increase of $\sigma_{2}$ (the front pod is on the step) and then its decrease (the rear pod is on the step). Its maximum value represents a configuration in which the central pod is on the top of the step and the two other pods are located on each side of it.

The last time interval $[12 s ; 14 s]$ represents the descent of the step. $\sigma_{2}$ is very strong during the descent of the central pod (figure 6). Then, when it is on the ground, $\sigma_{2}$ slowly decreases (figure 7), which means that the rear pod is in the phase of descent (figure 6), $\sigma_{1}$ takes again its maximum value after the obstacle clearance.

\section{CONCLusion}

This paper presents a general framework for evaluating wheeled modular vehicles composed of monocycles. We show that by using an equivalent kinematic model of a monocycle and the reciprocal screws theory, we derive easily the inverse velocity model that could be used for control and trajectory tracking. The force transmission in these systems is also investigated by making analogy to parallel manipulators and the concept of the manipulability ellipsoid. This theoretical study should be generalized to other vehicle kinematics including those with wheels, legs or both. Future works should be focused on minimization of torques and energy consumption during manoeuvring or steering along curved trajectory.

\section{ACKNOWLEDGMENT}

The authors would like to acknowledge DGA/SPART and ROBOSOFT for offering them the opportunity to develop a a scientific activity in the Miniroc framework.

\section{REFERENCES}

[1] http://www.robosoft.fr

[2] George M. Pierce, Robotics : Military applications for special operations forces, Research Report AU/ACSC/142/2000-04, Air Command and Staff College Air Uviversity, April 2000.

[3] P.F. Muir, C.P. Neumann, Kinematic modeling of wheeled mobile robots, Journal of Robotic Systems 4(2), pp 282-340 (1987).

[4] R. Rajagopalan, A generic kinematic formulation for wheeled mobile robots, Journal of Robotic Systems 14(2), pp 77-91 (1997).

[5] B.Y. Yi, W.K. Kim, The kinematics for redundantly actuated omnidirectional mobile robots, Journal of Robotic Systems 14(2), pp 255-267 (2002).

[6] M. Tarokh, G.J McDermott, Kinematics Modeling and Analyses of Articulated Rovers, IEEE Transactions on Robotics and Automation, Vol 21(4), pp539-553 (2005).

[7] K. Iagnemma, S. Dubowsky, Vehicle wheel/ground contact angle estimation : with application to mobile robot control, 7th International Symposium on Advances on Robot Kinematics, ARK'00, pp 137146,2000 .

[8] Ch. Grand, F. BenAmar, F. Plumet, and Ph. Bidaud, Stability and traction optimisation of high mobility rover - application to hybrid wheel-leg rover, The International Journal of Robotics Research, 23(1011):1041-1058, Oct. 2004

[9] W. K. Kim, S.E. Lee, B.J Yi, Mobility Analysis of Planar Mobile Robots, ICRA'2002 pp 2861-2867 (2002)

[10] L.A. Bonev, D. Zlatonov and C.M. Gosselin, Singularity analysis of 3 d.o.f planar parallel mechanisms via screw theory, Journal of Mechanical Engineering, vol. 125 pp 573-581, 2003.

[11] K.H. Hunt, Kinematic geometry of mechanims, Clarendon Press, 1978.

[12] D. S. Zlatanov, R. G. Fenton and B. Benhabib, Analysis of the Instantaneous Kinematics and Singular Configurations of Hybrid-Chain Manipulators, ASME 1994 Design Engineering Technical Conferences, DE-Vol. 72, pp. 467-476, September 11-14, Minneapolis. 